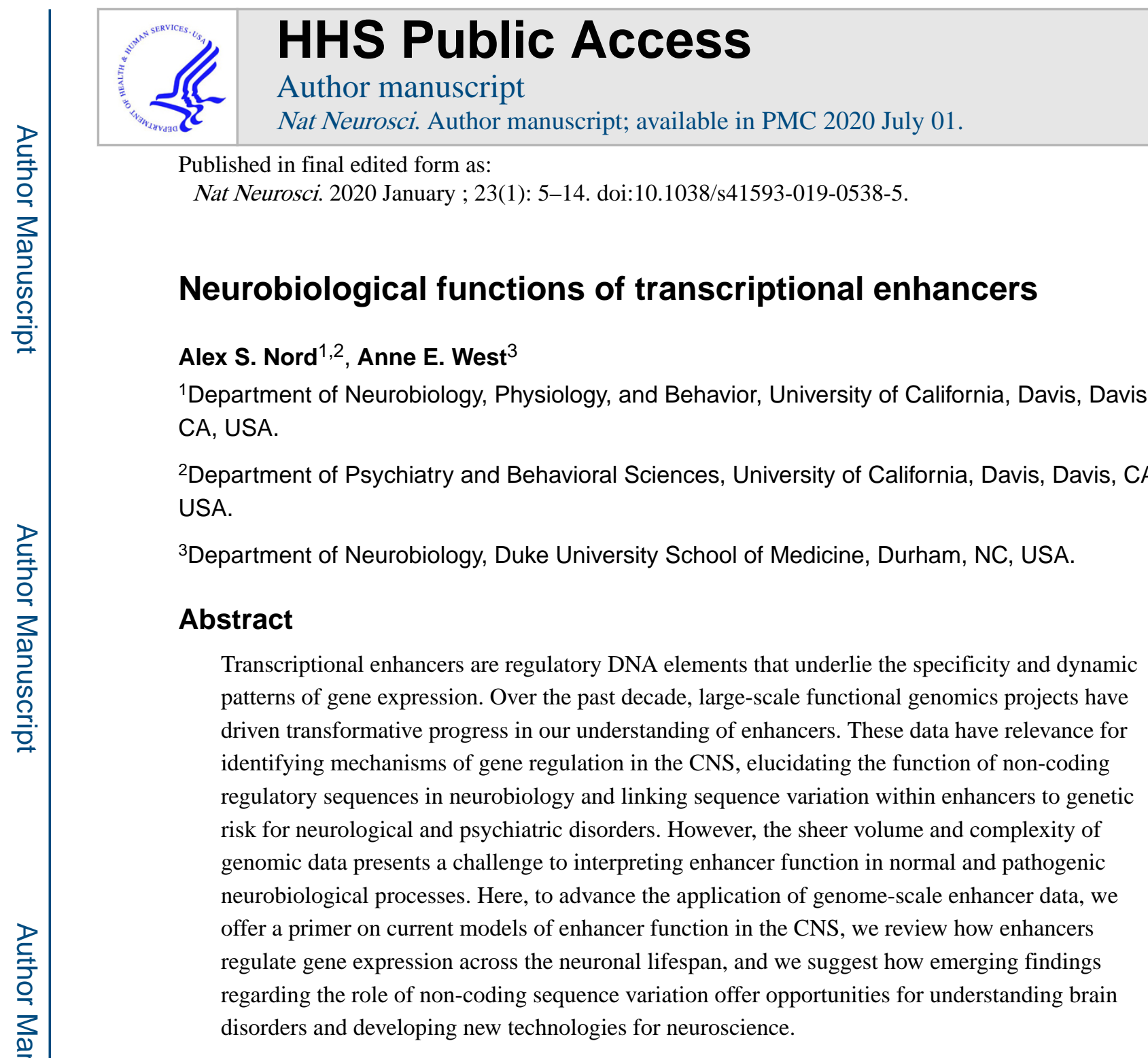

The human brain is arguably the most complex machine on Earth. The functions of the brain are the subject of our greatest fascinations, while the dysfunction of brain disorders represents a large and growing economic and societal burden. The genome encodes the rules for the construction and function of the brain, and it also harbors causes of disease susceptibility. The challenge for our post-genomic era is to decode how dynamic and celltype-specific genome function emerges from static sequence. Although the territory of the genome is vast, direct transcriptional control is concentrated in regulatory elements that comprise only a fraction of the total genome ${ }^{1}$, narrowing the search space. Enhancers are the most common regulatory elements in mammalian genomes, with hundreds of thousands of

\footnotetext{
Reprints and permissions information is available at www.nature.com/reprints.

Correspondence should be addressed to A.S.N. or A.E.W. asnord@ucdavis.edu; west@ neuro.duke.edu. Author contributions

A.S.N. and A.E.W. conceived of the review, researched the literature, wrote the manuscript and revised the manuscript.

Competing interests

The authors declare no competing interests.
}

Peer review information Nature Neuroscience thanks Erica Korb, Ian Maze, and the other, anonymous, reviewer(s) for their contribution to the peer review of this work.

Publisher's note Springer Nature remains neutral with regard to jurisdictional claims in published maps and institutional affiliations. 
enhancers predicted to coordinate transcriptional regulation in the brain ${ }^{2}$. Comparative analysis of these enhancer elements across CNS cell types and disease states holds clues to the basis of brain evolution, the wonders of cognition and the devastation of neurological disorders.

With fast, cheap, genome-wide sequencing methods available to profile regulatory genomes, consortium studies have targeted the brain as the next frontier. Data are already pouring in from large-scale projects including the BRAIN initiative cell census network ${ }^{3}$, which seeks to catalog all the cell types of the CNS, and PsychENCODE ${ }^{4}$, which seeks to elucidate the functional genomics of psychiatric disorders. From single-cell mRNA expression and epigenome mapping to multiplex chromatin modification landscapes to three-dimensional chromatin structure, sequencing-based functional genomics datasets are being generated from the CNS and deposited for public consumption at an astonishing rate. Will paradigmshifting findings emerge from these massive efforts? It is too soon to tell, but history suggests that these data will be the building blocks of a much more comprehensive story, with the widespread availability of regulatory genome maps seeding future advances in neuroscience that will go far beyond what we can envision now.

The goal of this review is to highlight emerging themes from CNS genome research that have the potential to transform neurobiology. First, we place emerging high-throughput enhancer mapping studies from CNS tissues in the context of the decades of mechanistic research on enhancer sequence, structure and function. We then summarize how enhancers define neuronal fate and coordinate gene expression across the long neuronal lifespan, and we review emerging data from human genetics studies that implicate enhancers in risk for brain disorders. Finally, we discuss outstanding questions and highlight future opportunities for studying enhancers in brain health and disease.

\section{What do we talk about when we talk about enhancers?}

The term 'enhancer' was originally applied only to DNA sequences that had demonstrated ability to increase gene expression when paired in cis with a core promoter ${ }^{5}$. However, the modern concept of the enhancer has evolved. This is because the explosion of methods for rapidly identifying elements with biochemical features of enhancers has fueled genomescale discovery of putative enhancers without directly interrogating function ${ }^{2}$. For example, based on the evidence that enhancers are mediators of cell-type differences in gene expression $^{6-8}$, descriptive proxies for enhancers such as histone modifications (Box 1) are now used to robustly complement and, in some cases, even replace expression data as a means to identify cell types in complex cellular mixtures ${ }^{9-11}$.

\section{What determines enhancer function?}

At the molecular level, an enhancer is a short stretch of DNA that provides docking sites for sequence-specific, DNA-binding transcription factors (TFs). The combinatorial binding of TFs determines the nature and extent of enhancer-mediated transcription ${ }^{12,13}$. The activity of cis-regulatory enhancer elements is therefore dependent upon the complement of TFs that are present in a given cell (i.e. the trans environment) (Fig. 1). This means that cell-type- 
specific differences in TF expression contribute to the cell-type-specific activity of different enhancer elements.

Yet most sequence-predicted TF binding sites are not occupied, even in cell types that express TFs with affinity for that sequence ${ }^{14}$. This is because local and regional native chromosomal context plays a major role in their function. Genomic DNA is packaged by histone proteins into repeating units called nucleosomes. TF binding requires clearance of histones from the binding site. This nucleosome displacement is detected biochemically by enzymatic methods that target histone-cleared regions of DNA ${ }^{15}$. Differences in enhancer accessibility between cells reflect differential TF binding and are among the strongest predictors of enhancer function in the endogenous context ${ }^{16}$. DNA methylation can also influence TF binding at enhancers by sterically blocking the binding of some TFs and/or by recruiting the binding of proteins that recognize methylated DNA ${ }^{17,18}$.

Three-dimensional chromosomal structure and regional epigenetic state also contribute to enhancer function and specificity. For example, deletion of chromosomal regions can create new enhancer-promoter interactions to induce new gene expression ${ }^{19-21}$. The position effects of chromatin structure are apparent from the evidence that extrachromosomal enhancer reporter assays can show ectopic activity compared with the function of the same regulatory elements in their endogenous chromosomal context ${ }^{22}$ and that the same enhancer can have various levels of activity depending on chromosomal insertion site ${ }^{23,24}$.

\section{What mechanisms underlie enhancer-modulated gene regulation?}

TFs at enhancers recruit chromatin-remodeling enzymes, structural proteins, transcriptional co-factors and RNA polymerase II (Pol II) onto their linked gene promoters (Fig. 2). The spatial interactions between enhancers and promoters are enabled by loops ${ }^{25}$ established via TF-dependent recruitment of chromatin-remodeling factors and structural proteins such as Mediator and $\mathrm{CTCF}^{26-28}$, which then increase recruitment and transcriptional activation of the Pol II complex. While many enhancers interact in cis with the nearest promoter, interaction mapping has shown that enhancers can also contact more distal promoters and that promoters of one gene can serve as enhancers for another ${ }^{29-31}$. Datasets are revealing enhancer-promoter interactions with cell-type, stage, region and stimulus specificity ${ }^{32,33}$. Nonetheless, it remains unclear exactly how much specificity exists between enhancers and promoters or even how enhancer-promoter interactions in general are actualized at the sequence, molecular and structural level ${ }^{34}$.

Biophysical models link enhancer-promoter interactions to more general properties associated with chromatin state and structure in the nucleus. Central to these models is a concept of stochastic interactions between enhancer and promoter elements that are stabilized by DNA-RNA-protein interactions and impacted by three-dimensional localization within the nucleus ${ }^{35-37}$. A recent model proposed that robust, consistent transcriptional activation associated with super-enhancers is mediated by highly interacting complexes that undergo a physical phase separation in the nucleus ${ }^{38}$. In comparison, loci that do not have such extensive biophysical interactions undergo sporadic transcriptional bursting ${ }^{39}$. Further determination of the properties of biophysical enhancer mechanisms in 
the brain will clarify the relationship between protein-DNA and DNA-DNA interactions in gene regulation.

At broader resolution, structural boundaries restrict enhancer- promoter interactions locally in topologically associated domains ${ }^{40}$, and enhancer-promoter contacts may have differential activity based on nuclear architecture. There is longstanding evidence of nuclear sites that are centers of transcription, often referred to as 'transcription factories' 41 . Conversely, localization to the lamina has been proposed to be a feature of chromosomal regions that are transcriptionally silenced ${ }^{42}$. Topologically associated domain regions can be annotated as a generally active state that is permissive of transcription or of the silenced state, providing higher-order control of enhancer activity and gene expression via physical partitioning within the nucleus ${ }^{40,43}$.

\section{How can we find CNS enhancers in the genome?}

The advent of high-throughput sequencing for functional genomic assays was a watershed moment, enabling genome-wide enhancer discovery using biochemical signatures (Box 1). The prediction of which sequences function as enhancers is enabled by a wide range of methodological approaches, including the mapping of open chromatin and DNA methylation; protein-DNA interactions to map histone marks, transcription factors or transcriptional co-activators such as p300; and chromosomal conformation mapping ${ }^{1,44}$ (Fig. 1). Inception of these methods enabled the field to move from a single enhancer or locus perspective to modeling the full regulatory genome of cells or tissues. In the past decade, the number of different methods of mapping transcriptional state, regulatory genomes and chromosomal contacts have blossomed and been widely applied to CNS research ${ }^{3,45}$. An ongoing revolution is further enabling the shift from analysis of bulk tissues and purified cells to single-cell and spatially resolved resolution of gene regulatory wiring in the developing and mature brain ${ }^{46-49}$.

Yet despite this outpouring of data, or perhaps because of it, there exists more than just an occasional lack of correspondence between the set of predicted enhancers and the ability of specific enhancers to drive expression in reporter assays ${ }^{50,51}$. At the level of any specific single locus, only some epigenomic signatures have high sensitivity for reporter-assayvalidated enhancer function, such as p300 in mouse brain ${ }^{2,52,53}$. There are multiple explanations for this disconnect, due to limitations both in sequence-based genome-wide methods (which lack direct functional testing) and in function-based methods (which lack endogenous chromatin context). Thus, significant gaps remain in our understanding of how enhancers actually work in endogenous genomic and epigenetic context.

The development of novel methods, such as via paired-protein-binding and chromosome contacts $^{54}$, massively parallel reporter assays ${ }^{55-57}$ and CRISPR-Cas9-mediated enhancer targeting ${ }^{58-61}$ has enabled the application of alternative, high-throughput approaches to function-based screening of enhancers. While these novel technologies have yet to be widely applied in the brain, they offer the promise of large-scale functional screening and the ability to test the sufficiency and necessity of individual enhancer sequences for gene regulation in CNS cells. 


\section{Enhancers orchestrate the timing of gene expression across the neuronal lifespan}

As is true for all cell types, enhancers contribute to neuronal fate determination. However, unlike many other cells, fate-committed neurons are specialized to have exceptionally long lives. Over this timespan, neurons must maintain the expression of genes that define the fate they adopted during development. At the same time, dynamic changes in gene expression are essential for adapting functional connectivity in the brain, both over the long term as neurons age and acutely in response to environmental stimuli ${ }^{62-64}$. The current challenge is to understand how enhancer activity is both dynamically regulated and stabilized over time (Fig. 3).

\section{Enhancers in cellular lineage specification and brain patterning}

Large-scale changes in enhancer usage have been shown to occur during early embryonic differentiation of neurons, for example during neural crest formation ${ }^{65}$ and in the transition from proliferative progenitors to post-mitotic neurons ${ }^{66}$. The developmental regulation of enhancer elements has also been documented during the differentiation of specific cell types, such as cerebellar granule cells ${ }^{67}$, cortical neural progenitors ${ }^{66}$ and motor neurons ${ }^{68}$. Comparison of the acetylation of histone H3 at lysine 27 (H3K27ac; Box 1) in mouse forebrain across seven developmental stages from embryonic day 11.5 to post-natal day 56 found that $90 \%$ of the putative enhancer loci show dynamic changes in H3K27ac across brain development ${ }^{69}$. A parallel pattern is present in the pseudotemporal developmental progression between germinal zone and cortical plate in fetal human cerebral cortex, where only a third of the putative regulatory regions showed static chromatin accessibility between regions ${ }^{66}$. Enhancer maps across cell types, developmental stages and brain regions are rapidly becoming available via the work of both individual labs and consortium efforts (Box 2). We highlight four key findings of these data.

First, most brain enhancers are active during select periods in development or in specific CNS cell types and brain subregions, consistent with the model that enhancers direct dynamic and differential gene expression patterns. The majority of such comparative studies are based on differential analysis of histone modifications and other biochemical signatures of enhancers (Box 1), but the limited functional enhancer testing in vivo in mouse suggests that stage-specific enhancers can indeed drive gene expression patterns associated with transient developmental processes in specific cells and regions of the embryonic brain ${ }^{52,69}$.

Second, although the activity of CNS enhancers is dependent on TF binding, the functional modes of these TFs are highly context-dependent, following rules regarding local epigenetic and biophysical states that remain to be fully understood. For example, detailed studies of individual TFs such as Nkx2.1 suggest that binding of even a single TF can play both activating and repressing roles depending on the binding site ${ }^{70}$. In addition, though many CNS TFs are predicted to bind widely across the genome, evidence from TF knockdown and knockout studies indicates that many of these events are dispensable for normal gene regulation ${ }^{70,71}$. A major goal for the field is to be able to predict TF-enhancer interactions and the functional impact of TF binding in the CNS, thus enabling reverse-engineering of 
the transcriptional control in the brain across the lifespan and in response to environmental and developmental cues.

Third, the lifespan of enhancer activity can be predicted by stereotyped changes in chromatin ${ }^{72,73}$. Initially, H3K4me1, along with $\mathrm{H} 3 \mathrm{~K} 27 \mathrm{me} 3$ at some enhancers, is present on poised or latent enhancers ${ }^{74,75}$. This is followed by recruitment of enzymes (for example, p300 and CBP) that deposit active histone marks (for example, H3K27ac and $\mathrm{H} 3 \mathrm{~K} 4 \mathrm{me} 3)^{53,69,76}$. Finally, enhancers undergo decommissioning back to latent or repressed states via activity of histone deacetylating and demethylating enzymes such as LSD1 (ref. ${ }^{77}$ ), as well as the regulation of DNA methylation ${ }^{78}$. These epigenomic changes not only reflect enhancer activity but also serve as a mechanism for regulating enhancer function, for example via restricting TF binding to enhancers via heterochromatin formation during neurodevelopment ${ }^{79}$.

Finally, pleiotropic functions of single enhancers and redundancy between multiple enhancers generate exceptional complexity in CNS regulatory genomes. For example, one single enhancer may act to drive expression of a target gene across similar or distinct cell types, either via distinct TF binding motifs or overlapping TF expression ${ }^{80}$. Most genes appear regulated by multiple enhancers even in the same cell type or developmental stage ${ }^{69,81}$, indicating that redundancy or overlapping activity is the norm rather than the exception. Thus, the often-used schematic featuring one enhancer driving each expression domain for a gene is a useful but vastly oversimplified representation of the complexity of CNS enhancer landscapes.

\section{Post-natal maturation of neuronal gene expression and the maintenance of cell fate}

The concept of 'terminal differentiation' takes on a new shade in cells like neurons, in which cell-cycle exit occurs so early in the overall cell lifespan. The full course of neuronal development consists not only of proliferation and differentiation but also migration, axon targeting, synaptic integration and functional maturation. Temporal profiling of gene expression in cortical regions of the macaque revealed that the adult gene expression profile fails to emerge until well into post-natal life ${ }^{82}$. This prolonged period of transcriptional maturation is functionally important in the brain in setting critical periods of sensorydependent cortical development ${ }^{62,83,84}$.

As in embryonic development, enhancers that increase their accessibility and/or their $\mathrm{H} 3 \mathrm{~K} 27 \mathrm{ac}$ association in post-natal neurons are strongly correlated with the increased expression of nearby genes ${ }^{67,85}$. Decreases in accessibility of specific enhancers are also pronounced during neuronal maturation and associated with decreased expression of nearby genes ${ }^{67,86}$. Interestingly, some enhancers are functionally decommissioned before becoming structurally inaccessible to TF binding ${ }^{76}$. This mechanism could underlie a period of differentiation plasticity, as has been observed in some cortical neuron types during early post-natal life ${ }^{67,87}$. Neuronal enhancer elements that lose accessibility over the course of brain development will eventually gain methylation of cytosines both at $\mathrm{CpG}$ dinucleotides (mCG) and at cytosines outside of the $\mathrm{CpG}$ context (mCH; Box 1) in the adult, which is 
thought to lock in their silenced state ${ }^{78}$. Determining the relative ordering of these chromatin changes at enhancers, and interfering with them experimentally using methods like local epigenome editing ${ }^{88}$, will advance understanding of how the epigenome orchestrates the process of neuronal maturation.

Given that enhancers define cell types, the dynamic nature of enhancer chromatin in maturing neurons raises the question of how fate is stably maintained in the face of these changes. Some TFs that serve as terminal selectors of fate remain expressed in fatecommitted neurons, suggesting their lifelong role in the maintenance of cell identity ${ }^{89}$. However, many of the TFs that establish cell identity are only transiently expressed, even though the terminal identity genes that they initiate remain expressed in the adult. This is the case in spinal motor neurons, in which stable expression of identity genes in maturing neurons is mediated by a distinct set of enhancers and TFs, compared with the mechanisms that regulate these same genes in development ${ }^{68}$. This evidence that dynamic changes in chromatin regulation lie beneath the seemingly constant transcription of genes may give clues to the timing and nature of sensitive periods for neuronal development.

\section{Enhancers in activity-dependent neuronal transcription}

In the brain, synaptic activity provides a salient stimulus for the adaptation of neuronal function through the induction of stimulus-dependent gene transcription ${ }^{90}$. Enhancers play both permissive and instructive roles in this process ${ }^{91}$. In their permissive role, the pattern of active enhancers predetermines which genes can be induced in any given kind of neuron ${ }^{92}$, such that distinct programs of activity-regulated gene expression are induced in different types of neurons, even in response to a common stimulus ${ }^{93,94}$. A functionally important corollary is that different types of neurons adapt in distinct ways to changes in neuronal firing 95 .

Enhancers are also direct targets of modulation by activity-induced signaling in neurons, and the resulting plasticity plays an instructive role in determining transcription ${ }^{96}$. Neuronal activity induces the expression from distal enhancers of short, bidirectional, non-coding RNAs (eRNAs) that are required for transcription to proceed ${ }^{97,98}$. eRNAs bind the negative elongation factor (NELF) to promote elongation at transcriptionally paused genes ${ }^{98}$. eRNAs can also bind to and modulate the function of the histone acetyltransferases p300 and CBP, which are recruited to neuronal enhancers that contain binding sites for activity-inducible transcription factors ${ }^{97,99}$. Activity-inducible recruitment of p300-CBP binding to activated $\mathrm{TFs}$ at enhancers is associated with a net increase in local $\mathrm{H} 3 \mathrm{~K} 27 \mathrm{ac}^{97,100}$. Enhancer acetylation modulates the bursting kinetics of activity-inducible genes, including Fos and Npas4, in ways that tune the magnitude of the transcriptional response to a given stimulus ${ }^{101}$. Acetylated histones can serve as docking sites for architectural factors such as Brd4 ${ }^{102}$. These proteins scaffold complexes that solidify promoter-enhancer interactions, which may explain the increased probability of promoter-enhancer looping observed in chromatin conformation assays following strong neuronal stimulation ${ }^{32,103}$ and could contribute to the physical repositioning of activated genes within the nucleus ${ }^{104}$. 
Finally, studies have shown that strong neuronal activity delivered in vivo by electrical stimulation of the dentate gyrus can increase or decrease the accessibility of regulatory elements in neurons, including enhancers ${ }^{105}$. Enhancers that showed increases in accessibility were enriched for the AP-1 binding site that is bound by TFs of the Fos-Jun family ${ }^{105}$. These data are consistent with the evidence that once it is inducibly expressed, Fos can recruit the chromatin remodeling protein Brg1 to its binding sites in the genome, providing a mechanism to clear histones from these enhancers ${ }^{106}$. Using Fos induction to change the landscape of enhancers available in a given cell could alter how neurons transcriptionally respond to subsequent stimuli, and thus is of interest as a potential mechanism of long-lasting neuronal plasticity.

\section{Enhancer dysfunction in brain disorders}

DNA mutation within enhancers can perturb the sequences or spacing of TF binding motifs or create new binding motifs that did not exist before. Thus, sequence variation in enhancers can result in decrease or loss of target gene expression in cells where gene expression is required or in ectopic expression in cell types where expression would not normally occur. Further, structural rearrangement via deletion, duplication or translocation can reposition enhancer sequences such that they target new genes. All of these enhancer-mediated mechanisms have been shown to contribute to brain disorders (Fig. 4).

\section{Enhancer sequence variation in brain disorders}

Rare disease-causing DNA variants that appear to solely impact enhancer function and gene regulation have been described, though only a few such cases involve brain disorders. For example, point mutation of non-coding sequence at a sonic hedgehog $(\mathrm{SHH})$ brain enhancer resulted in holoprosencephaly ${ }^{107}$, and non-coding deletions at the VIPR2 locus have been linked to schizophrenia ${ }^{108}$. While poorly understood, there are likely gene regulatory changes resulting from position effects in recurrent chromosomal microdeletions and microduplications. For example, RNA sequencing of mouse and in vitro models of recurrent $16 \mathrm{p} 11.2$ deletions and duplications found changes in gene regulation in the diploid genomic segments proximal to the copy number variants ${ }^{109}$.

The two major limitations of identifying rare pathogenic variants in enhancers are (i) the relatively small number of human genomes available for comparative sequence analysis and (ii) the difficulty of interpreting non-coding mutations. The first limitation is being rapidly remedied as population-level whole-genome sequencing (WGS) becomes standard, for example via the US National Institutes of Health (NIH) Precision Medicine Initiative ${ }^{110}$. These data enable testing for rare non-coding sequence variants with large effects on risk, with early reports in for cohorts with autism and intellectual disability ${ }^{111}$. The first studies investigating enhancer mutations have been mixed, and there is a lack of consensus in the field regarding the overall impact of rare regulatory mutations ${ }^{112,113}$. However, there is emerging statistical evidence for enrichment of de novo mutations in distal enhancers ${ }^{114}$ and in enhancers that are nearby promoters ${ }^{115}$ in simplex autism, in human accelerated regions in consanguineous autism spectrum disorder ${ }^{116}$ and for mutations in highly conserved fetal brain enhancers in developmental delay ${ }^{117}$. 
In comparison to rare mutations, there is strong evidence that common sequence variation at single-nucleotide polymorphisms (SNPs) in enhancer elements contributes across neuropsychiatric disorders. Robust and reproducible statistical associations have been reported across disorders including schizophrenia, bipolar disorder, major depressive disorder and autism spectrum disorder, with additional findings to come, for example via the Psychiatric Genetics Consortium ${ }^{118-122}$. The majority of genomic regions that are associated with disease risk have no plausible linked SNPs in coding sequences suggesting that the mechanism of disease lies within the non-coding regulatory genome. Indeed, many regions implicated by genome-wide association studies (GWAS) feature SNPs in putative enhancer sequences ${ }^{123,124}$.

\section{Linking regulatory variation to the neurobiology underlying brain disorders}

The challenge for both rare and common variation is shared: which DNA changes matter and why? For rare mutations, the first step will be identifying which of the many variations in any individual genome are causal. For common SNPs, the question is the same, but at least the search space is restricted to regions of linkage with the lead SNPs; even so, this can still include tens to hundreds of variants. At present, the majority of the work in this area involves fine mapping, expression quantitative trait loci (eQTL) mapping and improved in silico prediction to prioritize potential causal regulatory variants. For example, enhancerpromoter interactions mapped by $\mathrm{HiC}$ (three-dimensional genomic architecture) and eQTL mapping enable identification of how a regulatory SNP correlates with expression of a target gene ${ }^{125,126}$, and evolutionary conservation or TF binding site disruption can be used as filters to enrich for putative causal enhancer variants ${ }^{127}$.

A more ambitious challenge is determining how enhancer variation contributes to phenotype. The majority of functional studies of enhancer variation are limited to eQTL studies and ectopic assays in simple cellular models, which only assess whether a variant modulates transcription of a nearby gene in a reduced preparation. However, it is likely that disease-associated variants have context- and signaling-dependent impacts. For complex disorders, such as schizophrenia, it is possible that some aspects of risk originate in DNA variation, leading to different cell-type-specific or context-dependent enhancer activity. For example, thousands of enhancers have been mapped as involved in neuronal activitydependent transcriptional regulation, and sequence variants that disrupt this process would not necessarily be captured in bulk tissue or immortalized cell line assays. Unlike the identification of variants in populations, the functional testing of these variants is lowthroughput and requires the generation of time-intensive biological models and cellular phenotyping.

Where individual enhancer variants have been studied, results reinforce the structural concepts of enhancer function we summarized above. One example is the first intron of CACNA1C, which encodes an L-type calcium channel and contains a linkage block with SNPs associated with schizophrenia and bipolar disorder. Some of these SNPs fall within regions predicted to be enhancers, and when compared with the non-disease associated common variant in an enhancer reporter assay, the disease SNP conferred altered enhancer activity on the reporter plasmid ${ }^{45}$. In another example, SNPs in the glucocorticoid regulatory 
FKBP5 gene that are associated with stress-associated psychiatric disorders were found to alter the strength of interaction of the FKBP5 promoter with distal regulatory elements ${ }^{128}$. The net result of this structural disruption is enhanced stress-dependent induction of FKBP5 in disease-susceptible individuals, leading to long term dysregulation of the stress hormone axis. A third example is a noncoding variant on $7 \mathrm{p} 21$ associated with frontotemporal lobar degeneration that influences CTCF-mediated long-range chromatin looping interactions between cis-regulatory regions and the promoter of the TMEM106B gene ${ }^{129}$. Looping increases the expression of TMEM106B protein, which disrupts lysosomal function and increases cell toxicity.

\section{Conclusions and future directions}

The function of enhancers is dependent on sequence and context, and changes to both enhancer sequence (for example, mutations) and context (for example, epigenetic response) are associated with CNS disorders. With so much data already at hand, how will this knowledge seed future progress and what challenges remain?

\section{Filling in the blank spots in the cartography of CNS enhancers}

While major progress has been made in identifying enhancers at the global level, existing maps of CNS regulatory genomes lack the granularity required to understand the regulation of many specific genes of interest. Nonetheless, these datasets are already being used for tool development, such as the generation of enhancer-driven viruses to drive cell-typespecific transgene expression ${ }^{130}$ and to elucidate the role of enhancers in CNS evolution ${ }^{131}$. There is hope the field will soon have access to relatively complete sets of predicted enhancer activity maps at high spatial, temporal and cell-type resolution for the CNS. Enhancer activity predictions will need to be paired with chromosomal interaction maps to reveal target genes and nuclear DNA structure. To accomplish this, technical limitations must be overcome with regard to chromosomal resolution and analysis of rare or limited cell populations. Genome sequencing of extant and extinct organisms ${ }^{132-135}$, paired with population efforts such as the 1000 Genome Project ${ }^{136}$, are enabling the dissection of enhancer evolutionary history and population genetics. In parallel, eQTL studies have begun to pair sequence variation and gene expression for healthy and disease-state CNS tissues $^{137,138}$.

\section{Reverse engineering the brain, from mapping to mechanisms}

There is critical need to address the blind spot between genome-wide predictions and ectopic reporter assays toward building a mechanistic understanding of enhancers in the CNS. A first step will be understanding how the complex enhancer landscapes that genomic technologies have revealed act across development to produce tightly regulated gene expression patterns. A second step will be to reveal the combinatorial activities of enhancers as they function in an endogenous chromatin and nuclear context.

For example, a recent study combined ectopic enhancer assays, enhancer deletion mouse studies and single-cell RNA sequencing to resolve the necessity and sufficiency of individual 
enhancers that activate expression of the Arx transcription factor. For a set of two enhancers that are active in dorsal forebrain and two enhancers active in ventral forebrain in early embryonic brain development, this study found that deletion of any one single enhancer caused a reduction in Arx expression in specific cell types, whereas paired deletions of either the dorsal or ventral enhancers caused more severe phenotypes ${ }^{58}$. In a conceptually parallel study, the stimulus-specific responsiveness of individual Fos enhancers was mapped, elucidating physiological specificity of activity-dependent Fos transcriptional induction ${ }^{32}$ and suggesting a path toward more sensitive enhancer-based readouts of neural activity. At present, work dissecting transcriptional circuitry for even a single gene requires extensive resources, and advances in technology will be needed to make such detailed studies of single-gene transcriptional regulation feasible genome-wide.

With the development of CRISPR-Cas9-based approaches, it has become more feasible to model and modulate endogenous enhancers. A recent effort showed proof-of-principle for enhancer-mediated transcriptional modulation as a treatment paradigm in the context of Sim 1 haploinsufficiency-associated obesity in mouse ${ }^{139}$. In this work, a synthetic transcriptional activator based on inactivated Cas9 was targeted via guide RNA to an endogenous enhancer that drives Sim1 expression in the hypothalamus. The study showed that via this enhancer-mediated molecular intervention, Sim1 expression could be rescued to wild-type levels and obesity prevented. Other efforts are underway to use enhancers as targets for synthetic transcriptional activators or repressors and to use enhancer sequences in conditional expression and gene therapy vectors to fine tune exogenous expression constructs.

\section{Enhancers as handles for understanding CNS disorders and brain evolution}

Well-powered GWAS and emerging WGS have identified non-coding intervals and variants linked to major brain disorders from autism to Alzheimer's disease, building a map of regulatory variant risk burden for CNS disorders. Enhancer activity differences between healthy and disease states can also give information regarding non-genetic features of brain disorders. For example, patterns that have already been identified include enrichment of neuroimmune-relevant enhancers identified via GWAS on schizophrenia and Alzheimer's or enrichment of de novo mutations from autism cases near genes active in fetal brain development ${ }^{118,140,141}$. A small number of studies have begun to characterize chromatin from the adult brain, comparing normal aging with neurodegenerative disease states or models, mainly focusing on profiling histone modifications ${ }^{142-145}$. These are examples of epigenome-wide association studies, and further application of this strategy offers the opportunity to discover the general role of enhancers as mediators signaling pathways and environmental challenges associated with brain disorders.

By understanding where and when relevant enhancers are active, there is the potential not only to understand causal biological processes, but also to localize such biology to specific cell types, developmental stages or environmental challenge. In the long term, such information may lead to precision drug development and will be paired with polygenic risk scores or WGS mutation profiles to guide diagnosis and treatment. The same logic holds for understanding the impacts of regulatory sequence variation associated with human brain 
evolution, with comparative enhancer maps now available across brain development and evolutionary lineages to enable understanding of how changes to enhancers in the human genome are associated with changes in development, morphology and function of the human brain.

Fully realizing these goals of a well-developed understanding of enhancer biology in the CNS will not be easy. Unlike the primary DNA sequence of the genome, the regulatory function underwritten by enhancers is highly dynamic and will be specific to each cell type, developmental stage, context and disease state. However, the tools already exist to address most of the major challenges regarding mapping and annotating enhancer elements in the CNS, with new technologies certain to arise in the coming years.

\section{Acknowledgements}

This work was supported by NIH R35GM119831 (A.S.N.) and R01NS098804 (A.E.W.).

\section{References}

1. Kellis $\mathrm{M}$ et al. Defining functional DNA elements in the human genome. Proc. Natl. Acad. Sci. USA 111, 6131-6138 (2014). [PubMed: 24753594]

2. ENCODE Project Consortium. An integrated encyclopedia of DNA elements in the human genome. Nature 489, 57-74 (2012). [PubMed: 22955616]

3. Ecker JR et al. The BRAIN Initiative Cell Census Consortium. The BRAIN Initiative Cell Census Consortium: lessons learned toward generating a comprehensive brain cell atlas. Neuron 96, 542 557 (2017). [PubMed: 29096072]

4. PsychENCODE Consortium. Revealing the brain's molecular architecture. Science 362, 1262-1263 (2018). [PubMed: 30545881]

5. Banerji J, Rusconi S \& Schaffner W Expression of a beta-globin gene is enhanced by remote SV40 DNA sequences. Cell 27, 299-308 (1981). [PubMed: 6277502]

6. Voss SD, Schlokat U \& Gruss P The role of enhancers in the regulation of cell-type-specific transcriptional control. Trends Biochem. Sci 11, 287-289 (1986).

7. O'Kane CJ \& Gehring WJ Detection in situ of genomic regulatory elements. Drosoph. Proc. Natl. Acad. Sci. USA 84, 9123-9127 (1987).

8. Song L et al. Open chromatin defined by DNaseI and FAIRE identifies regulatory elements that shape cell-type identity. Genome Res. 21, 1757-1767 (2011). [PubMed: 21750106]

9. Cao J et al. Joint profiling of chromatin accessibility and gene expression in thousands of single cells. Science 361, 1380-1385 (2018). [PubMed: 30166440]

10. Cusanovich DA et al. The cis-regulatory dynamics of embryonic development at single-cell resolution. Nature 555, 538-542 (2018). [PubMed: 29539636]

11. Cao J et al. Comprehensive single-cell transcriptional profiling of a multicellular organism. Science 357, 661-667 (2017). [PubMed: 28818938]

12. Wang $\mathrm{J}$ et al. Sequence features and chromatin structure around the genomic regions bound by 119 human transcription factors. Genome Res. 22, 1798-1812 (2012). [PubMed: 22955990]

13. Inukai S, Kock KH \& Bulyk ML Transcription factor-DNA binding: beyond binding site motifs. Curr. Opin. Genet. Dev 43, 110-119 (2017). [PubMed: 28359978]

14. Hombach D, Schwarz JM, Robinson PN, Schuelke M \& Seelow D A systematic, large-scale comparison of transcription factor binding site models. BMC Genomics 17, 388 (2016). [PubMed: 27209209]

15. Becker PB \& Workman JL Nucleosome remodeling and epigenetics. Cold Spring Harb. Perspect. Biol 5, a017905 (2013). [PubMed: 24003213] 
16. Thurman RE et al. The accessible chromatin landscape of the human genome. Nature $489,75-82$ (2012). [PubMed: 22955617]

17. Maurano MT et al. Role of DNA methylation in modulating transcription factor occupancy. Cell Rep. 12, 1184-1195 (2015). [PubMed: 26257180]

18. Zuo Z, Roy B, Chang YK, Granas D \& Stormo GD Measuring quantitative effects of methylation on transcription factor-DNA binding affinity. Sci. Adv 3, o1799 (2017).

19. Lettice LA et al. Enhancer-adoption as a mechanism of human developmental disease. Hum. Mutat 32, 1492-1499 (2011). [PubMed: 21948517]

20. Lupiáñez DG et al. Disruptions of topological chromatin domains cause pathogenic rewiring of gene-enhancer interactions. Cell 161, 1012-1025 (2015). [PubMed: 25959774]

21. Lupiáñez DG, Spielmann M \& Mundlos S Breaking TADs: how alterations of chromatin domains result in disease. Trends Genet. 32, 225-237 (2016). [PubMed: 26862051]

22. Bird AP \& Wolffe AP Methylation-induced repression-belts, braces, and chromatin. Cell 99, 451454 (1999). [PubMed: 10589672]

23. Nord AS Learning about mammalian gene regulation from functional enhancer assays in the mouse. Genomics 106, 178-184 (2015). [PubMed: 26079655]

24. Maricque BB, Chaudhari HG \& Cohen BA A massively parallel reporter assay dissects the influence of chromatin structure on cis-regulatory activity. Nat. Biotechnol 37, 90-95 (2018).

25. Levine M, Cattoglio C \& Tjian R Looping back to leap forward: transcription enters a new era. Cell 157, 13-25 (2014). [PubMed: 24679523]

26. Kuras L, Borggrefe T \& Kornberg RD Association of the Mediator complex with enhancers of active genes. Proc. Natl. Acad. Sci. USA 100, 13887-13891 (2003). [PubMed: 14623974]

27. Ren $\mathrm{G}$ et al. CTCF-mediated enhancer-promoter interaction is a critical regulator of cell-to-cell variation of gene expression. Mol. Cell 67, 1049-1058.e6 (2017). [PubMed: 28938092]

28. Whyte WA et al. Master transcription factors and mediator establish super-enhancers at key cell identity genes. Cell 153, 307-319 (2013). [PubMed: 23582322]

29. Zhang Y et al. Chromatin connectivity maps reveal dynamic promoter-enhancer long-range associations. Nature 504, 306-310 (2013). [PubMed: 24213634]

30. Nolis IK et al. Transcription factors mediate long-range enhancer-promoter interactions. Proc. Natl. Acad. Sci. USA 106, 20222-20227 (2009). [PubMed: 19923429]

31. Diao $Y$ et al. A tiling-deletion-based genetic screen for cis-regulatory element identification in mammalian cells. Nat. Methods 14, 629-635 (2017). [PubMed: 28417999]

32. Joo JY, Schaukowitch K, Farbiak L, Kilaru G \& Kim TK Stimulus-specific combinatorial functionality of neuronal c-fos enhancers. Nat. Neurosci. 19, 75-83 (2016). [PubMed: 26595656]

33. Mitchell AC et al. Longitudinal assessment of neuronal 3D genomes in mouse prefrontal cortex. Nat. Commun 7, 12743 (2016). [PubMed: 27597321]

34. van Arensbergen J, van Steensel B \& Bussemaker HJ In search of the determinants of enhancerpromoter interaction specificity. Trends Cell Biol. 24, 695-702 (2014). [PubMed: 25160912]

35. Hnisz D, Shrinivas K, Young RA, Chakraborty AK \& Sharp PA A phase separation model for transcriptional control. Cell 169, 13-23 (2017). [PubMed: 28340338]

36. Sigova AA et al. Transcription factor trapping by RNA in gene regulatory elements. Science 350 , 978-981 (2015). [PubMed: 26516199]

37. Chen $\mathrm{H}$ et al. Dynamic interplay between enhancer-promoter topology and gene activity. Nat. Genet 50, 1296-1303 (2018). [PubMed: 30038397]

38. Sabari BR et al. Coactivator condensation at super-enhancers links phase separation and gene control. Science 361, eaar3958 (2018). [PubMed: 29930091]

39. Bothma JP et al. Dynamic regulation of Eve Stripe 2 expression reveals transcriptional bursts in living Drosophila embryos. Proc. Natl. Acad. Sci. USA 111, 10598-10603 (2014). [PubMed: 24994903]

40. Dixon JR et al. Topological domains in mammalian genomes identified by analysis of chromatin interactions. Nature 485, 376-380 (2012). [PubMed: 22495300]

41. Sutherland H \& Bickmore WA Transcription factories: gene expression in unions? Nat. Rev. Genet 10, 457-466 (2009). [PubMed: 19506577] 
42. Guelen L et al. Domain organization of human chromosomes revealed by mapping of nuclear lamina interactions. Nature 453, 948-951 (2008). [PubMed: 18463634]

43. Dowen JM et al. Control of cell identity genes occurs in insulated neighborhoods in mammalian chromosomes. Cell 159, 374-387 (2014). [PubMed: 25303531]

44. Heintzman ND et al. Distinct and predictive chromatin signatures of transcriptional promoters and enhancers in the human genome. Nat. Genet 39, 311-318 (2007). [PubMed: 17277777]

45. Akbarian S et al. The PsychENCODE project. Nat. Neurosci 18, 1707-1712 (2015). [PubMed: 26605881]

46. Luo C et al. Single-cell methylomes identify neuronal subtypes and regulatory elements in mammalian cortex. Science 357, 600-604 (2017). [PubMed: 28798132]

47. Preissl S et al. Single-nucleus analysis of accessible chromatin in developing mouse forebrain reveals cell-type-specific transcriptional regulation. Nat. Neurosci 21, 432-439 (2018). [PubMed: 29434377]

48. Lake BB et al. Integrative single-cell analysis of transcriptional and epigenetic states in the human adult brain. Nat. Biotechnol 36, 70-80 (2018). [PubMed: 29227469]

49. Wang $X$ et al. Three-dimensional intact-tissue sequencing of single-cell transcriptional states. Science 361, eaat5691 (2018). [PubMed: 29930089]

50. Kwasnieski JC, Fiore C, Chaudhari HG \& Cohen BA High-throughput functional testing of ENCODE segmentation predictions. Genome Res. 24, 1595-1602 (2014). [PubMed: 25035418]

51. Inoue F \& Ahituv N Decoding enhancers using massively parallel reporter assays. Genomics 106, 159-164 (2015). [PubMed: 26072433]

52. Visel A et al. A high-resolution enhancer atlas of the developing telencephalon. Cell 152, 895-908 (2013). [PubMed: 23375746]

53. Visel A et al. ChIP-seq accurately predicts tissue-specific activity of enhancers. Nature 457, $854-$ 858 (2009). [PubMed: 19212405]

54. Mumbach MR et al. HiChIP: efficient and sensitive analysis of protein-directed genome architecture. Nat. Methods 13, 919-922 (2016). [PubMed: 27643841]

55. Shen SQ et al. Massively parallel cis-regulatory analysis in the mammalian central nervous system. Genome Res 26, 238-255 (2016). [PubMed: 26576614]

56. Grossman SR et al. Positional specificity of different transcription factor classes within enhancers. Proc. Natl. Acad. Sci. USA 115, E7222-E7230 (2018). [PubMed: 29987030]

57. Nguyen TA et al. High-throughput functional comparison of promoter and enhancer activities. Genome Res. 26, 1023-1033 (2016). [PubMed: 27311442]

58. Dickel DE et al. Ultraconserved enhancers are required for normal development. Cell 172, 491499.e15 (2018). [PubMed: 29358049]

59. Xie S, Duan J, Li B, Zhou P \& Hon GC Multiplexed engineering and analysis of combinatorial enhancer activity in single cells. Mol. Cell 66, 285-299.e5 (2017). [PubMed: 28416141]

60. Canver MC et al. BCL11A enhancer dissection by Cas9-mediated in situ saturating mutagenesis. Nature 527, 192-197 (2015). [PubMed: 26375006]

61 . Huang J et al. Dissecting super-enhancer hierarchy based on chromatin interactions. Nat. Commun 9, 943 (2018). [PubMed: 29507293]

62. Chen LF, Zhou AS \& West AE Transcribing the connectome: roles for transcription factors and chromatin regulators in activity-dependent synapse development. J. Neurophysiol 118, 755-770 (2017). [PubMed: 28490640]

63. Robison AJ \& Nestler EJ Transcriptional and epigenetic mechanisms of addiction. Nat. Rev. Neurosci 12, 623-637 (2011). [PubMed: 21989194]

64. Yap EL \& Greenberg ME Activity-regulated transcription: bridging the gap between neural activity and behavior. Neuron 100, 330-348 (2018). [PubMed: 30359600]

65. Prescott SL et al. Enhancer divergence and cis-regulatory evolution in the human and chimp neural crest. Cell 163, 68-83 (2015). [PubMed: 26365491]

66. de la Torre-Ubieta $\mathrm{L}$ et al. The dynamic landscape of open chromatin during human cortical neurogenesis. Cell 172, 289-304.e18 (2018). [PubMed: 29307494] 
67. Frank CL et al. Regulation of chromatin accessibility and Zic binding at enhancers in the developing cerebellum. Nat. Neurosci 18, 647-656 (2015). [PubMed: 25849986]

68. Rhee HS et al. Expression of terminal effector genes in mammalian neurons is maintained by a dynamic relay of transient enhancers. Neuron 92, 1252-1265 (2016). [PubMed: 27939581]

69. Nord AS et al. Rapid and pervasive changes in genome-wide enhancer usage during mammalian development. Cell 155, 1521-1531 (2013). [PubMed: 24360275]

70. Sandberg M et al. Transcriptional networks controlled by NKX2-1 in the development of forebrain GABAergic neurons. Neuron 91, 1260-1275 (2016). [PubMed: 27657450]

71. Cusanovich DA, Pavlovic B, Pritchard JK \& Gilad Y The functional consequences of variation in transcription factor binding. PLoS Genet. 10, e1004226 (2014). [PubMed: 24603674]

72. Calo E \& Wysocka J Modification of enhancer chromatin: what, how, and why? Mol. Cell 49, 825837 (2013). [PubMed: 23473601]

73. Spicuglia S \& Vanhille L Chromatin signatures of active enhancers. Nucleus 3, 126-131 (2012). [PubMed: 22555596]

74. Rada-Iglesias A et al. A unique chromatin signature uncovers early developmental enhancers in humans. Nature 470, 279-283 (2011). [PubMed: 21160473]

75. Creyghton MP et al. Histone H3K27ac separates active from poised enhancers and predicts developmental state. Proc. Natl. Acad. Sci. USA 107, 21931-21936 (2010). [PubMed: 21106759]

76. Russ BE et al. Regulation of $\mathrm{H} 3 \mathrm{~K} 4 \mathrm{me} 3$ at transcriptional enhancers characterizes acquisition of virus-specific $\mathrm{CD}^{+}{ }^{+} \mathrm{T}$ cell-lineage-specific function. Cell Rep 21, 3624-3636 (2017). [PubMed: 29262339]

77. Whyte WA et al. Enhancer decommissioning by LSD1 during embryonic stem cell differentiation. Nature 482, 221-225 (2012). [PubMed: 22297846]

78. Lister R et al. Global epigenomic reconfiguration during mammalian brain development. Science 341, 1237905 (2013). [PubMed: 23828890]

79. Zhu J et al. Genome-wide chromatin state transitions associated with developmental and environmental cues. Cell 152, 642-654 (2013). [PubMed: 23333102]

80. Preger-Ben Noon E et al. Comprehensive analysis of a cis-regulatory region reveals pleiotropy in enhancer function. Cell Rep. 22, 3021-3031 (2018). [PubMed: 29539428]

81. Wenger AM et al. The enhancer landscape during early neocortical development reveals patterns of dense regulation and co-option. PLoS Genet. 9, e1003728 (2013). [PubMed: 24009522]

82. Bakken TE et al. A comprehensive transcriptional map of primate brain development. Nature 535, 367-375 (2016). [PubMed: 27409810]

83. Roberts AC et al. Downregulation of NR3A-containing NMDARs is required for synapse maturation and memory consolidation. Neuron 63, 342-356 (2009). [PubMed: 19679074]

84. Sheng M, Cummings J, Roldan LA, Jan YN \& Jan LY Changing subunit composition of heteromeric NMDA receptors during development of rat cortex. Nature 368, 144-147 (1994). [PubMed: 8139656]

85. Thakurela S, Sahu SK, Garding A \& Tiwari VK Dynamics and function of distal regulatory elements during neurogenesis and neuroplasticity. Genome Res. 25, 1309-1324 (2015). [PubMed: 26170447]

86. Daum JM et al. The formation of the light-sensing compartment of cone photoreceptors coincides with a transcriptional switch. eLife 6, e31437 (2017). [PubMed: 29106373]

87. Rouaux C \& Arlotta P Direct lineage reprogramming of post-mitotic callosal neurons into corticofugal neurons in vivo. Nat. Cell Biol 15, 214-221 (2013). [PubMed: 23334497]

88. Holtzman L \& Gersbach CA Editing the epigenome: reshaping the genomic landscape. Annu. Rev. Genomics Hum. Genet 19, 43-71 (2018). [PubMed: 29852072]

89. Deneris ES \& Hobert O Maintenance of postmitotic neuronal cell identity. Nat. Neurosci 17, 899907 (2014). [PubMed: 24929660]

90. Lyons MR \& West AE Mechanisms of specificity in neuronal activity-regulated gene transcription. Prog. Neurobiol 94, 259-295 (2011). [PubMed: 21620929] 
91. Gray JM et al. Genomic views of transcriptional enhancers: essential determinants of cellular identity and activity-dependent responses in the CNS. J. Neurosci 35, 13819-13826 (2015). [PubMed: 26468181]

92. Whitney $\mathrm{O}$ et al. Core and region-enriched networks of behaviorally regulated genes and the singing genome. Science 346, 1256780 (2014). [PubMed: 25504732]

93. Mardinly AR et al. Sensory experience regulates cortical inhibition by inducing IGF1 in VIP neurons. Nature 531, 371-375 (2016). [PubMed: 26958833]

94. Hrvatin S et al. Single-cell analysis of experience-dependent transcriptomic states in the mouse visual cortex. Nat. Neurosci 21, 120-129 (2018). [PubMed: 29230054]

95. Spiegel I et al. Npas4 regulates excitatory-inhibitory balance within neural circuits through celltype-specific gene programs. Cell 157, 1216-1229 (2014). [PubMed: 24855953]

96. Madabhushi R \& Kim TK Emerging themes in neuronal activity-dependent gene expression. Mol. Cell. Neurosci 87, 27-34 (2018). [PubMed: 29254824]

97. Kim TK et al. Widespread transcription at neuronal activity-regulated enhancers. Nature 465, 182 187 (2010). [PubMed: 20393465]

98. Schaukowitch K et al. Enhancer RNA facilitates NELF release from immediate early genes. Mol. Cell 56, 29-42 (2014). [PubMed: 25263592]

99. Bose DA et al. RNA binding to CBP stimulates histone acetylation and transcription. Cell 168, 135-149.e22 (2017). [PubMed: 28086087]

100. Malik AN et al. Genome-wide identification and characterization of functional neuronal activitydependent enhancers. Nat. Neurosci 17, 1330-1339 (2014). [PubMed: 25195102]

101. Chen LF et al. Enhancer histone acetylation modulates transcriptional bursting dynamics of neuronal activity-inducible genes. Cell Rep. 26, 1174-1188.e5 (2019). [PubMed: 30699347]

102. Zippo A et al. Histone crosstalk between H3S10ph and H4K16ac generates a histone code that mediates transcription elongation. Cell 138, 1122-1136 (2009). [PubMed: 19766566]

103. Yamada $\mathrm{T}$ et al. Sensory experience remodels genome architecture in neural circuit to drive motor learning. Nature 569, 708-713 (2019). [PubMed: 31068695]

104. Walczak A et al. Novel higher-order epigenetic regulation of the Bdnf gene upon seizures. J. Neurosci 33, 2507-2511 (2013). [PubMed: 23392678]

105. Su Y et al. Neuronal activity modifies the chromatin accessibility landscape in the adult brain. Nat. Neurosci 20, 476-483 (2017). [PubMed: 28166220]

106. Vierbuchen $\mathrm{T}$ et al. AP-1 transcription factors and the BAF complex mediate signal-dependent enhancer selection. Mol. Cell 68, 1067-1082.e12 (2017). [PubMed: 29272704]

107. Jeong Y et al. Regulation of a remote Shh forebrain enhancer by the Six 3 homeoprotein. Nat. Genet 40, 1348-1353 (2008). [PubMed: 18836447]

108. Vacic V et al. Duplications of the neuropeptide receptor gene VIPR2 confer significant risk for schizophrenia. Nature 471, 499-503 (2011). [PubMed: 21346763]

109. Blumenthal I et al. Transcriptional consequences of $16 \mathrm{p} 11.2$ deletion and duplication in mouse cortex and multiplex autism families. Am. J. Hum. Genet 94, 870-883 (2014). [PubMed: 24906019]

110. Collins FS \& Varmus H A new initiative on precision medicine. N. Engl. J. Med 372, 793-795 (2015). [PubMed: 25635347]

111. Yuen C \& K. Ret al. Whole genome sequencing resource identifies 18 new candidate genes for autism spectrum disorder. Nat. Neurosci 20, 602-611 (2017). [PubMed: 28263302]

112. Werling DM et al. An analytical framework for whole-genome sequence association studies and its implications for autism spectrum disorder. Nat. Genet 50, 727-736 (2018). [PubMed: 29700473]

113. Bryois $\mathrm{J}$ et al. Evaluation of chromatin accessibility in prefrontal cortex of individuals with schizophrenia. Nat. Commun 9, 3121 (2018). [PubMed: 30087329]

114. Turner TN et al. Genomic patterns of de novo mutation in simplex autism. Cell 171, 710-722.e12 (2017). [PubMed: 28965761]

115. An JY et al. Genome-wide de novo risk score implicates promoter variation in autism spectrum disorder. Science 362, eaat6576 (2018). [PubMed: 30545852] 
116. Doan RN et al. Mutations in human accelerated regions disrupt cognition and social behavior. Cell 167, 341-354.e12 (2016). [PubMed: 27667684]

117. Short PJ et al. De novo mutations in regulatory elements in neurodevelopmental disorders. Nature 555, 611-616 (2018). [PubMed: 29562236]

118. Schizophrenia Working Group of the Psychiatric Genomics Consortium. Biological insights from 108 schizophrenia-associated genetic loci. Nature 511, 421-427 (2014). [PubMed: 25056061]

119. Yu D et al. Interrogating the genetic determinants of Tourette's syndrome and other tic disorders through genome-wide association studies. Am. J. Psychiatry 176, 217-227 (2019). [PubMed: 30818990]

120. Grove $\mathrm{J}$ et al. Identification of common genetic risk variants for autism spectrum disorder. Nat. Genet 51, 431-444 (2019). [PubMed: 30804558]

121. Howard DM et al. Genome-wide meta-analysis of depression identifies 102 independent variants and highlights the importance of the prefrontal brain regions. Nat. Neurosci 22, 343-352 (2019). [PubMed: 30718901]

122. Demontis D et al. Discovery of the first genome-wide significant risk loci for attention deficit/ hyperactivity disorder. Nat. Genet 51, 63-75 (2019). [PubMed: 30478444]

123. Roussos P et al. A role for noncoding variation in schizophrenia. Cell Rep. 9, 1417-1429 (2014). [PubMed: 25453756]

124. Gusev A et al. Partitioning heritability of regulatory and cell-type-specific variants across 11 common diseases. Am. J. Hum. Genet 95, 535-552 (2014). [PubMed: 25439723]

125. Won $\mathrm{H}$ et al. Chromosome conformation elucidates regulatory relationships in developing human brain. Nature 538, 523-527 (2016). [PubMed: 27760116]

126. Rajarajan P et al. Neuron-specific signatures in the chromosomal connectome associated with schizophrenia risk. Science 362, eaat4311 (2018). [PubMed: 30545851]

127. Visscher PM et al. 10 years of GWAS discovery: biology, function, and translation. Am. J. Hum. Genet 101, 5-22 (2017). [PubMed: 28686856]

128. Klengel T et al. Allele-specific FKBP5 DNA demethylation mediates gene-childhood trauma interactions. Nat. Neurosci 16, 33-41 (2013). [PubMed: 23201972]

129. Gallagher MD et al. A dementia-associated risk variant near TMEM106B alters chromatin architecture and gene expression. Am. J. Hum. Genet 101, 643-663 (2017). [PubMed: 29056226]

130. Dimidschstein $\mathrm{J}$ et al. A viral strategy for targeting and manipulating interneurons across vertebrate species. Nat. Neurosci 19, 1743-1749 (2016). [PubMed: 27798629]

131. McLean CY et al. Human-specific loss of regulatory DNA and the evolution of human-specific traits. Nature 471, 216-219 (2011). [PubMed: 21390129]

132. Lewin HA et al. Earth BioGenome Project: sequencing life for the future of life. Proc. Natl. Acad. Sci. USA 115, 4325-4333 (2018). [PubMed: 29686065]

133. Meyer $\mathrm{M}$ et al. A high-coverage genome sequence from an archaic Denisovan individual. Science 338, 222-226 (2012). [PubMed: 22936568]

134. Prüfer K et al. The complete genome sequence of a Neanderthal from the Altai Mountains. Nature 505, 43-49 (2014). [PubMed: 24352235]

135. McCoy RC, Wakefield J \& Akey JM Impacts of Neanderthal-introgressed sequences on the landscape of human gene expression. Cell 168, 916-927.e12 (2017). [PubMed: 28235201]

136. 1000 Genomes Project Consortium, Auton A et al. A global reference for human genetic variation. Nature 526, 68-74 (2015). [PubMed: 26432245]

137. Wang D et al. Comprehensive functional genomic resource and integrative model for the human brain. Science 362, eaat8464 (2018). [PubMed: 30545857]

138. Dobbyn A et al. Landscape of conditional eQTL in dorsolateral prefrontal cortex and colocalization with schizophrenia GWAS. Am. J. Hum. Genet 102, 1169-1184 (2018). [PubMed: 29805045]

139. Matharu N et al. CRISPR-mediated activation of a promoter or enhancer rescues obesity caused by haploinsufficiency. Science 363, eaau0629 (2019). [PubMed: 30545847]

140. Jansen IE et al. Genome-wide meta-analysis identifies new loci and functional pathways influencing Alzheimer's disease risk. Nat. Genet 51, 404-413 (2019). [PubMed: 30617256] 
141. Amiri A et al. Transcriptome and epigenome landscape of human cortical development modeled in organoids. Science 362, eaat6720 (2018). [PubMed: 30545853]

142. Berson A, Nativio R, Berger SL \& Bonini NM Epigenetic regulation in neurodegenerative diseases. Trends Neurosci. 41, 587-598 (2018). [PubMed: 29885742]

143. Klein HU et al. Epigenome-wide study uncovers large-scale changes in histone acetylation driven by tau pathology in aging and Alzheimer's human brains. Nat. Neurosci 22, 37-46 (2019). [PubMed: 30559478]

144. Nativio R et al. Dysregulation of the epigenetic landscape of normal aging in Alzheimer's disease. Nat. Neurosci 21, 497-505 (2018). [PubMed: 29507413]

145. Gjoneska $\mathrm{E}$ et al. Conserved epigenomic signals in mice and humans reveal immune basis of Alzheimer's disease. Nature 518, 365-369 (2015). [PubMed: 25693568] 


\section{Box 1}

\section{Functional genomics approaches for enhancer characterization}

\section{Open chromatin:}

One of the most efficient ways to find putative enhancers using high-throughput sequencing is to generate maps of open chromatin regions. Genomic elements where TFs are bound lack nucleosomes, and thus these regions are preferentially digested by DNaseI or micrococcal nuclease and more easily fragmented via sonication. These cut regions of the genome can be recovered, sequenced and mapped back on the genome. More recently, direct transposase-mediated labeling of open chromatin (ATAC-seq) has streamlined the method, allowing it to be applied down to single-cell resolution. Nonetheless, open chromatin is a non-specific indicator of the function of any given regulatory element, because other elements such as promoters and insulators (along with enhancers) also feature this signature.

\section{DNA-protein interactions:}

Chromatin immunoprecipitation followed by sequencing (ChIP-seq) is the most common approach to mapping both TF-DNA interactions and the local enrichment of histone modifications across the genome. DNA-binding proteins are usually first cross-linked to genomic DNA, and the DNA is sheared into small pieces by mechanical sonication or enzymatic digestion. Then, highly specific antibodies directed against a DNA-binding protein or post-translationally modified histone are used to precipitate the co-associated DNA fragments.

\section{Histone modifications:}

Post-translational modifications of the N-terminal tails of histone proteins are correlated with the functional state of the associated chromatin and thus are considered to comprise what is known as the 'histone code'. These histone modifications, which include acetylation, methylation and phosphorylation at specific amino acids, can easily be mapped genome-wide by ChIP-seq and thus serve as descriptive proxies for the function of the underlying DNA regulatory elements. Active enhancers are marked by the acetylation of histone $\mathrm{H} 3$ at lysine 27 (H3K27ac), monomethylation at lysine 4 (H3K4me1), and binding of the H3K27 histone acetyltransferases CBP or p300. Regions that bear H3K4me1 but lack H3K27ac are considered 'poised' enhancers, because at least some of these elements will gain $\mathrm{H} 3 \mathrm{~K} 27 \mathrm{ac}$ and become active enhancers in response to developmental or environmental cues.

\section{DNA methylation:}

Active enhancers can be demarcated by differential methylation, primarily of cytosine residues in $\mathrm{CpG}$ dinucleotides ( $\mathrm{mCG}$ ). Though less common, cytosines in genomic DNA from post-natal neurons can also be modified by methylation when they are found in $\mathrm{CpA}$ (most common), $\mathrm{CpC}$, and $\mathrm{CpT}$ dinucleotides, which collectively are referred to as $\mathrm{mCH}$ sites. Methylation can be assayed via multiple approaches, including bisulfite sequencing or antibody-based pulldown. 


\section{Transcription of enhancers:}

With the recognition that enhancers appear to be frequently transcribed contemporaneously with their linked gene promoters, RNA-sequencing approaches have been used to map not only the location but also the activity of enhancers. Because most eRNAs are unstable, methods that can capture newly transcribed or nascent RNA increase the sensitivity of detection.

\section{Chromosomal interactions:}

Chromosomal interaction mapping can identify nuclear DNA interactions, including enhancer-promoter contacts. These methods traditionally rely on enzymatic ligation of regions that are in close physical proximity in the nucleus. Chromosomal contacts can be detected either by unbiased genome-wide profiling (for example, Hi-C) or via selection of enhancer-relevant interactions with a DNA-binding protein, in which immunoprecipitation is paired with interaction assays (for example, ChIA-PET).

\section{Enhancer mutagenesis:}

Mutagenesis remains the gold standard for functional genomics, with engineered Cas9 proteins capable of generating double-stranded DNA breaks (DSBs) at specific DNA sequences via guide RNA (gRNA) targeting providing a useful complement to traditional transgenic strategies. Cas9 can be used to modify the sequences of endogenous enhancer regions via introduction of mutations through non-homologous end joining and other error-prone cellular mechanisms for DSB repair, via deletion of sequences between Cas9 target sites and via introduction of specific alleles through homology-based repair with a DNA template. While mutagenesis is possible within any cell where the Cas9 constructs can be delivered, allelic knock-in remains restricted to cells that undergo homologydirected repair and is not currently feasible in post-mitotic CNS cells.

\section{Epigenome editing:}

More rapid, though less sequence-specific analysis of enhancer function can be derived from the local recruitment of enzymatically dead Cas9 (dCas9) fused with chromatin regulators such as KRAB or p300 that inhibit (CRISPRi) or activate (CRISPRa) regulatory elements. CRISPR-dCas9-based assays can be used to interrogate single enhancers, or guide RNAs targeting multiple enhancers can be multiplexed for highthroughput screening. 


\section{Box 2 |}

\section{Bioinformatic enhancer resources for neuroscience}

Genome-wide chromosome interaction, epigenomic and transcriptomic methods for enhancer annotation have been fruitfully applied across individual studies and in large centralized efforts. These studies have revealed the presence of hundreds of thousands of cis-regulatory DNA elements with putative enhancer function in brains of flies, mice and humans. Many of the centralized efforts have built online interfaces for these datasets, generating valuable bioinformatics resources for neuroscientists.

- $\quad$ ENCODE (https://www.encodeproject.org/): transcriptomics and epigenomics from CNS tissues across humans and model organisms across development

- $\quad$ Roadmap Epigenomics Project (http://www.roadmapepigenomics.org/): epigenomics from various human fetal and adult CNS tissues

- $\quad$ FANTOM (http://fantom.gsc.riken.jp/5/): eRNA mapping for mammalian CNS tissues

- GTEx (https://gtexportal.org/home/): gene expression and SNP genotyping for eQTL mapping for human CNS tissues

- $\quad$ VISTA Enhancer Browser (https://enhancer.lbl.gov/): in vivo enhancer data for embryonic mouse brain

- BRAIN Initiative Cell Census Network (https://biccn.org/): transcriptomics and epigenomics for specific CNS tissues and cells from developing and adult mouse and human brain

- PsychENCODE (http://resource.psychencode.org/): transcriptomics and epigenomics from healthy and CNS disorder tissues and in vitro models

- CommonMind Consortium (https://www.synapse.org/cmc): data relevant to neuropsychiatric disorders 


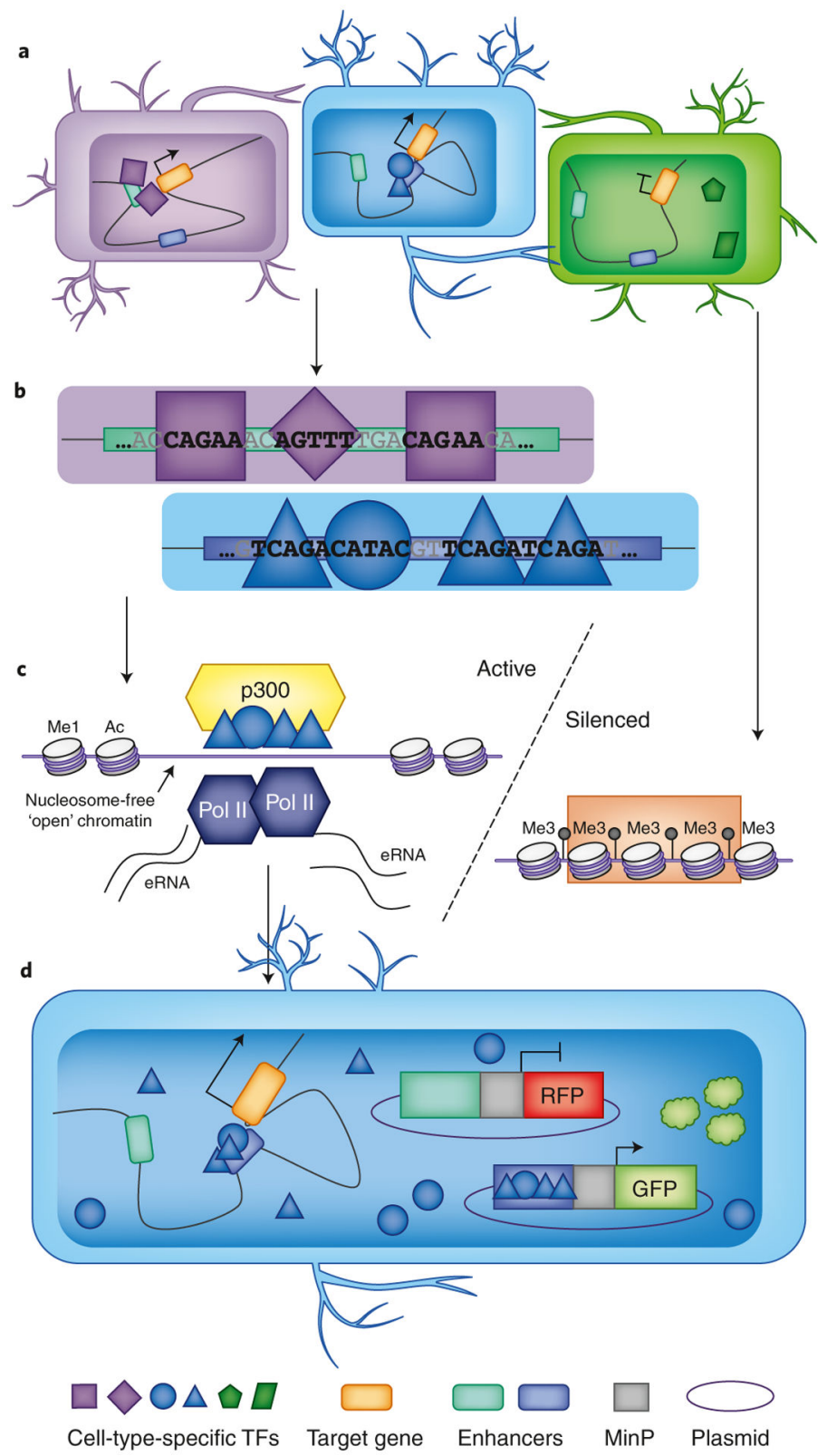

Fig. 1 |. Enhancer function is dependent on sequence and context.

a, Schematic of three neuronal cell types in the CNS illustrating a simple model of enhancermediated gene expression. Gene $X$ is expressed in two of the three cell types and, in this simplified schematic expression, is mediated by one cell-type-specific enhancer in each cell. The activity of each enhancer is modulated by transcription factors that have cell-typespecific expression patterns. TF binding enables transcriptional activation via establishing enhancer-promoter physical interactions and recruiting co-factors and transcriptional complexes. b, TF binding is based on DNA-binding domains that have the ability to bind to specific sequence motifs. TF interaction is dependent on affinity to specific sequence motifs, with potential different regulatory impacts based on binding affinity. TF interaction can also be dependent on combinatorial binding or interactions between TFs; for example, one TF 
may not bind its cognitive recognition sequence unless a second TF is also bound. c, Context-dependent epigenetics impact enhancer activity, and enhancers can be identified by characteristic patterns of biochemical interaction and chromatin state. Left: an active enhancer, characterized by TF binding (blue triangles and circles), co-activators interaction (p300 shown as example), nucleosome-free open chromatin, characteristic histone posttranslational modifications (H3K27ac and H3K4me1 are two of the most common marks), RNA Pol II complex recruitment and localized bidirectional eRNA transcription. Right: an enhancer that is epigenetically silenced, characterized by DNA methylation (black circles), histone PTMs such as H3K27me3, and corepressors such as polycomb repressor complex (PCR; orange square). d, The canonical definition of an enhancer is based on sequenceencoded function. Enhancers are capable of driving expression in a bidirectional, contextindependent manner, generally as established via ectopic enhancer-reporter assays. In these assays, the candidate enhancer is cloned upstream of a minimal promoter and reporter gene (for example, GFP or RFP) and the construct is delivered to cells. In this example, when delivered to Cell B, Enhancer 2 drives expression of the reporter, whereas Enhancer 1 does not, due to which cell-type-specific TFs are present in Cell B. minP, minimal promoter. 

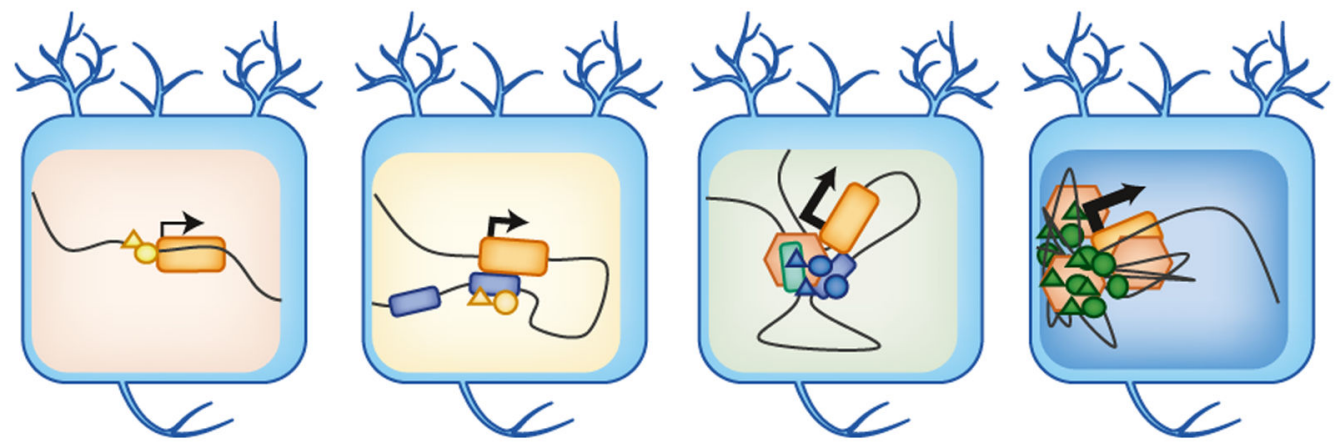
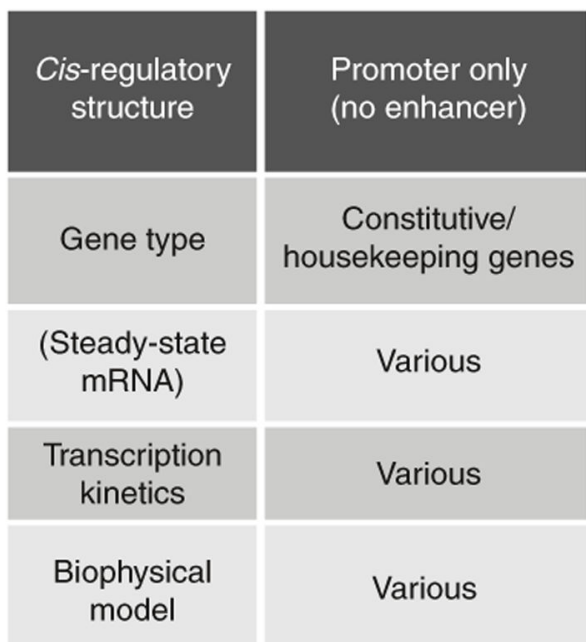

Constitutive/ housekeeping genes

\begin{tabular}{|l|}
\hline Various \\
\hline Various \\
\hline Various \\
\hline
\end{tabular}

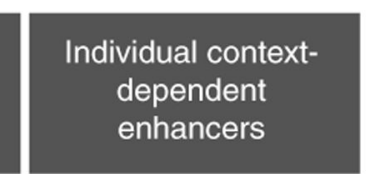

\section{Specialized and context-dependent genes}

Low to medium

Bursting

\section{Stochastic, low to} medium frequency RNA Pol II recruitment

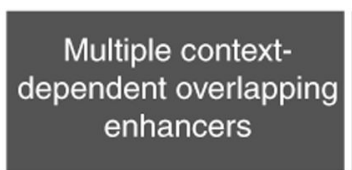

Specialized and context-dependent genes

Medium to high

Bursting

\section{Stochastic, medium \\ to high frequency RNA Pol II recruitment}

\section{'Super' enhancer \\ (many clustered \\ enhancers) \\ Lineage- determination genes \\ High \\ Constitutive \\ Phase-shift, constant RNA PollI recruitment}

Fig. 2 |. General models of CNS regulatory wiring.

Different general cis-regulatory structures exist across gene types in the CNS. Images represent simplified schematic of cis-regulatory structure and biophysical interactions mediating transcriptional state, with summary of cis-regulatory structure, gene type, steadystate mRNA concentration, transcription kinetics and biophysical components summarized in the table below. 


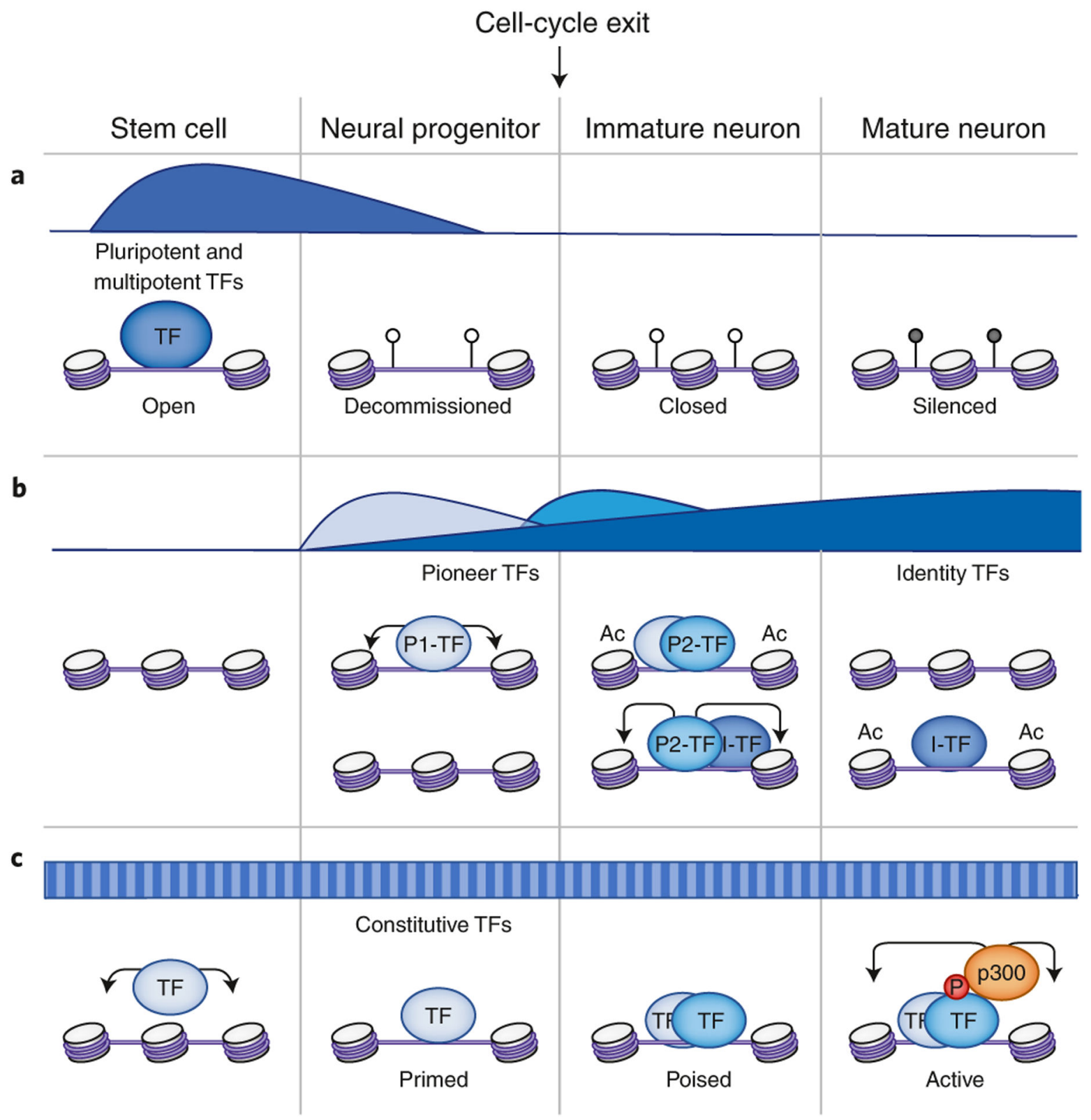

Fig. $3 \mid$. Enhancers across the neuronal lifespan.

Expression patterns of stage-dependent TFs are indicated over time. The effects of these TFs on enhancer accessibility and activity are indicated by the position of histones, the binding of TFs and modifications of DNA (lollipops; white, unmethylated; black, methylated) and histones (methylation, me1; acetylation, ac). a, Pluripotency TFs are rapidly downregulated when progenitors commit to the neuronal fate, and the enhancers they regulate are first decommissioned then permanently silenced. $\mathbf{b}$, Identity TFs (ITF) for specific neuronal fates can act as pioneer factors ( $\mathrm{P} 1$ - and $\mathrm{P} 2-\mathrm{TF})$ to open chromatin at enhancers that regulate neuronal cell-type-specific genes. Some of these TFs are downregulated after fate commitment, and other TFs will take their place. However, some identity TFs continue to be expressed and play a role either in fate maintenance and/or switch their targets to promote 
maturation. c, Constitutively expressed TFs can promote stage-specific gene expression through their signal-dependent modification and state-specific recruitment of transcriptional co-activators like p300-CBP. 
a
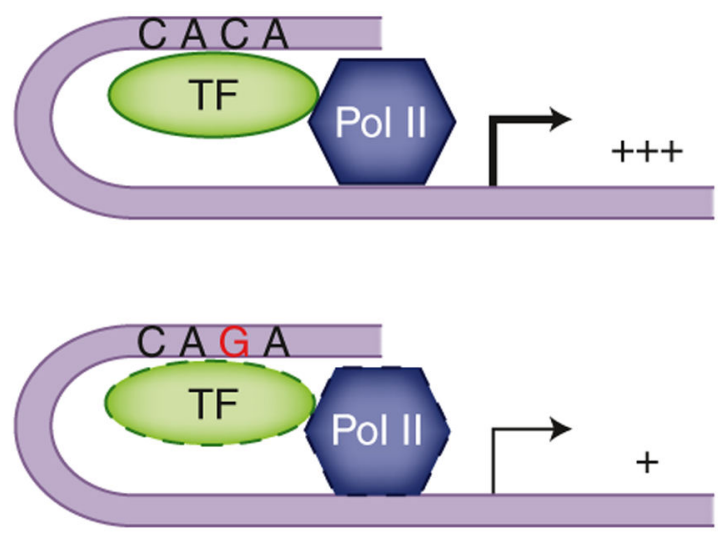

b

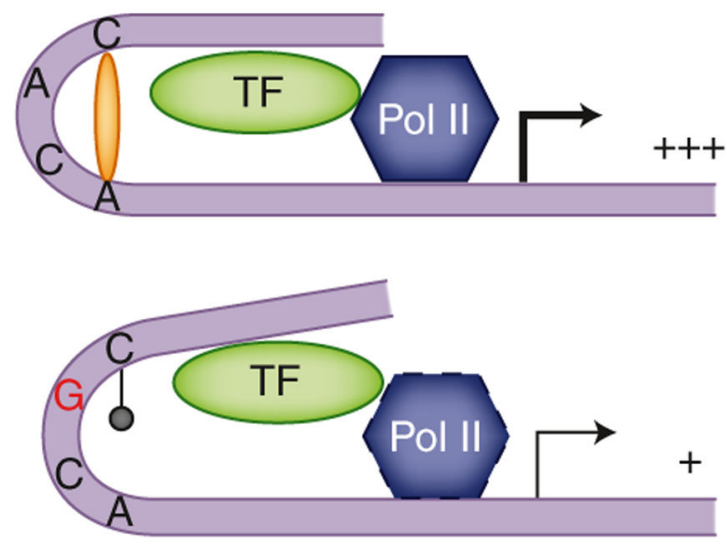

C

\section{In vivo}

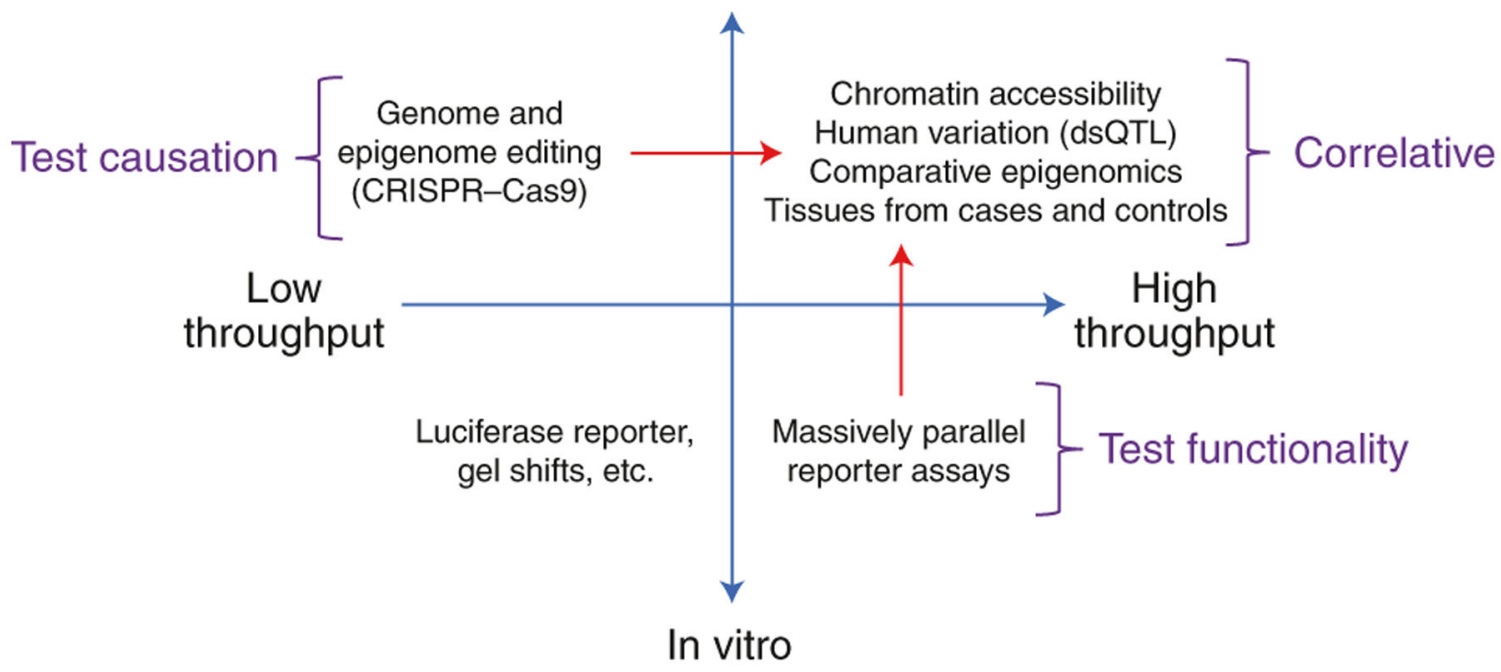

Fig. 4 |. How disease-associated SNPs impact enhancer function.

a, Sequence variants may occur within the TF binding sites of an enhancer. In this case, the change in sequence can decrease or increase the binding affinity of the recruited TF (indicated by dotted lines), impacting the extent of enhancer-driven expression of coupled genes. b, SNPs may also occur between the coupled enhancer and its target gene promoter. In the example shown, a $\mathrm{C}$ is part of a binding site for an architectural factor (tan oval), and when the neighboring nucleotide changes to $\mathrm{G}$ from $\mathrm{A}$, methylation (black lollipop) of the $\mathrm{C}$ blocks recruitment of the architectural factor. In this case the impact of the variation is likely to be structural, affecting the strength or specificity of promoter-enhancer looping, and reducing Pol II recruitment to coupled gene promoters. The thin arrows and single + represent low levels of transcription, whereas the thick arrows and +++ represent higher levels of transcription. c, Strategies for determining which non-coding sequence variants have consequences for disease phenotypes. The application of high-throughput functional validation of elements and CRISPR-Cas9 editing to the in vivo setting offers high potential 
for functionally meaningful validation. The red arrows highlight leading-edge advances that are bringing CRISPR-Cas9 and reporter assays toward the high-throughput in vivo category. 